Natural Hazards and Earth System Sciences (2003) 3: 217-228

(C) European Geosciences Union 2003

Natural Hazards and Earth System Sciences

\title{
Experience of short term earthquake precursors with VLF-VHF electromagnetic emissions
}

\author{
K. Eftaxias, P. Kapiris, J. Polygiannakis, A. Peratzakis, J. Kopanas, G. Antonopoulos, and D. Rigas \\ Department of Physics, University of Athens, Panepistimiopolis, Zografos, 15784 Athens, Greece
}

Received: 6 June 2002 - Revised: 24 July 2002 - Accepted: 26 August 2002

\begin{abstract}
Electromagnetic anomalies (EMA) covering a wide range of frequencies from ULF, VLF up to VHF have been observed before recent destructive earthquakes in continental Greece. We show that the features of these signals are possibly correlated with the fault model characteristics of the associated earthquake and with the degree of geotectonic heterogeneity within the focal zone. The time evolution of these electromagnetic sequences reveals striking similarities to that observed in laboratory acoustic and electromagnetic emissions during different stages of failure preparation process in rocks. If we consider that the same dynamics governs the large-scale earthquakes and the microscopic scale sample rheological structure, the results of this analysis suggest that the recorded EMA might reflect the nucleation phase of the associated impending earthquake. We focus on the rise of the statistical view of earthquakes. We find electromagnetic fingerprints of an underlying critical mechanism. Finally, we conclude that it is useful to combine ULF and VLF-VHF field measurements in an attempt to enhance the understanding of the physics behind these observations and thus to improve the quality of earthquake prediction. Further, the identification of an EMA as a seismogenic one supports the characterization of a sequence of shocks as foreshocks at the time they occur, further helping the earthquake prediction effort.
\end{abstract}

\section{Introduction}

Short-term earthquake prediction is one of the most challenging targets in Earth science today. Many recent reports have presented examples from several regions of the world by which associations between possible electromagnetic precursors and earthquakes become more and more plausible (Varotsos and Alexopoulos, 1984a; Varotsos and Alexopoulos, 1984b; Gokhberg et al., 1995; Hayakawa, and Fuji-

Correspondence to: K. Eftaxias

(ceftax@phys.uoa.gr) nawa, 1994; Lighthill, 1996; Biagi, 1999; Hayakawa, 1999; Hayakawa et al., 2000; Uyeda, 2000; Morgounov, 2001; Nagao et al., 2001; Telesca et al., 2001; Gershenzon and Bambakidis, 2001; Hayakawa and Molchanov, 2002). The 1995 $M=7.2$ Kobe event was a big impact, because pre-seismic electromagnetic changes in different frequency ranges from DC to VHF were detected by scientists who were largely working independently and in isolation (Nagao et al., 2001). It was probably the first case where multiple methods detected possible precursors for one earthquake. However, a number of scientists believe that there is still no definite proof that electromagnetic signals and pre-seismic process in the Earth crust are connected. Each major earthquake raises the question as to whether or not it was preceded by some phenomena that could be considered as precursory. Thus, more data on the presence of electromagnetic signatures of earthquakes need to be accumulated.

An important characteristic of probable electromagnetic precursors is their appearance in a wide frequency band, covering DC-ULF, VLF and LF to VHF ranges, possibly involving many different processes. The time sequence deduced from these various observations seems to qualitatively agree with the idea that different frequency EMA are not synchronous; signals of the DC-ULF band tend to appear earlier (Tate and Daily, 1989; Qian et al., 1994; Uyeda, 1996). DCULF EM signals have a precursory time from few days to several weeks (Varotsos et al., 1996; Hayakawa et al., 1999, 2000 ) in contrast to the much shorter lead-time (from few to several hours) for the VLF-VHF signals (Gokhberg et al., 1982; Warwick et al., 1982; Oike and Ogawa, 1982; Oike and Yamada, 1994; Vershinin, 1999; Eftaxias et al., 2001b, 2002). A challenging prospective could be the possibility to estimate the parameters (location, time, and magnitude) of the forthcoming earthquake from independent precursory data sets, detected at different time periods, such as the DCULF and VLF-VHF signals. The achievement of converging estimations would definitely improve the chances of accurate prediction.

The reliability of earthquake forecasts depends on how 
well the model, on which the forecast is based, fits the actual processes in the crust. Whether EM precursors to EQs exist or not is an important question for not only EQ but also for understating the physical processes of EQ generation. The modelling of EQ precursors is a fundamental tool for understanding the physical processes in fault zones before seismic rupture. Our target for the near future is the comprehensive understanding of the physics behind these observations. The above-mentioned two groups of EMA may arise from different mechanisms since they do not occur with the same time scales. It seems useful, as strongly recommended by Hayakawa et al. (1994), to combine DC-ULF and VLFVHF field measurements to enhance the understanding of the physics behind these observations and to improve the quality of the EQ prediction.

We mainly focus on VLF-VHF signals. Warwick et al. (1982) and Gokhberg et al. (1982) are pioneering examples for this category of electromagnetic precursory phenomena. Soon it was found that the $1-100 \mathrm{kHz}$ frequency range is informative for investigation of the final stage of destruction (Morgounov, 2001). This is reasonable due to their short precursory time.

\section{Observational system}

At the end of 1994, a station to monitor EMA was installed in the mountain area of Zante island $\left(37.76^{\circ} \mathrm{N}-20.76^{\circ} \mathrm{E}\right)$ in Western Greece. The station consists of: (i) six loop antennas detecting the three components (EW, NS and vertical) of the variation of the magnetic field at $3 \mathrm{kHz}$ and $10 \mathrm{kHz}$, (ii) two $\lambda / 2$ type electric dipoles detecting the electric field variations at $41 \mathrm{MHz}$ and $54 \mathrm{MHz}$ and (iii) two Short Thin Wire Antennas (STWA), detecting the electric field variations at DCULF frequency band, oriented at EW and NS directions of a length of $100 \mathrm{~m}$, respectively. A STWA sensor is a simple insulated dipole antenna lying on the Earth's surface (Thiel, 1978). The $3,10 \mathrm{kHz}$ and $41,54 \mathrm{MHz}$ frequencies were selected to minimize the effects of the sources of cultural noise. All the electromagnetic time-series were sampled at $1 \mathrm{~Hz}$. Such an experimental set-up helps to specify not only whether or not a single VLF or VHF electromagnetic disturbance is preseismic in itself, but also whether a combination of such disturbances at different frequency bands, could be characterized as preseismic according to accumulated laboratory and field experience (Eftaxias et al., 2000, 2002). The station was operating during the following two time periods: December 1994 to December 1995 and September 1998 to date.

\section{Observation results}

From 1995 to 1999 the heavily destructive earthquakes which occurred in continental Greece were: (i) The 13 May 1995 Kozani-Grevena (K-G) earthquake in NW Greece $\left(40.2^{\circ} \mathrm{N}-\right.$ $21.7^{\circ} \mathrm{E}$ ) with a magnitude $M_{s}(\mathrm{ATH})=6.6$ and focal depth of $11 \mathrm{~km}$ (Clarke et al., 1997). (ii) The 15 June 1995 EgionEratini earthquake in central Greece $\left(38.4^{\circ} \mathrm{N}-22.1^{\circ} \mathrm{E}\right)$ with $M_{s}(\mathrm{ATH})=6.5$. (iii) The 7 September 1999 Athens earthquake $\left(38.2^{\circ} \mathrm{N}-23.6^{\circ} \mathrm{E}\right)$ with a magnitude $M_{s}(\mathrm{ATH})=5.9$ and focal depth of $17 \mathrm{~km}$ (Papadopoulos, 2002). No surface faulting was observed (Kontoes et al., 2000). All three earthquakes were preceded by strong EMA recorded at Zante station at different frequency bands (Eftaxias et al., 2000, 2001a, b, 2002).

\section{EMA detected prior to the Kozani-Grevena earth- quake}

EMA observed before the Kozani-Grevena (K-G) earthquake were in the following time order: (i) Approximately thirty hours prior to the K-G earthquake, EMA at the high frequency bands of $41 \mathrm{MHz}$ and $54 \mathrm{MHz}$ were recorded. They ceased approximately $1 \mathrm{~h}$ before the EQ occurrence (Fig. 1a). Note that the high frequency part of seismo-electromagnetic signals emanating from the source region is strongly absorbed by the crust. For a homogeneous half-space of $\rho=$ $4 \cdot 10^{4} \Omega \cdot \mathrm{m}$ and for frequencies of the order of a few $\mathrm{MHz}$, the skin depth is $\sim 10 \mathrm{~m}$. In the case of the K-G earthquake, the observed clear fault traces of NE-SW direction and of (8-12) km length (Meyer et al., 1998) could justify the appearance of the $\mathrm{MHz}$ signals. With this explanation in mind, the absence of VHF signals in a series of pre-seismic disturbances is reasonable; at the same time, this absence of VHF EMA provides a useful piece of information, namely, that the pending EQ will not precipitate ruptures at the Earth's surface. (ii) At the tail of the $41 \mathrm{MHz}$ and $54 \mathrm{MHz}$ EMA, strong multi-peaked EM signals of duration approximately $30 \mathrm{~min}$, with both sharp onsets and ends, emerged at $3 \mathrm{kHz}$ and $10 \mathrm{kHz}$. These signals also ceased approximately one hour before the earthquake (Fig. 1b). We draw the attention to the almost simultaneous cessation of anomalies at both $\mathrm{MHz}$ and $\mathrm{kHz}$ frequency bands. (iii) During the daytime on 12 May 1995, strong EMA of duration of a few hours were also recorded on the EW/NS STWA dipoles. The regular daily behaviour was broken down; there was a clear inversion of the usual daily variations with higher frequency components (Fig. 1c). The features of the detected anomalies are compatible with those reported by other authors (e.g. Qian et al., 1994).

\section{EMA detected prior to Athens earthquake}

Very clear EMA have been detected in the VLF band (Fig. 2), i.e., at $3 \mathrm{kHz}$ and $10 \mathrm{kHz}$, before the Athens EQ (Eftaxias et al., 2000, 2001b). The whole electromagnetic precursors were emerged from 31 August to 7 September 1999 (Fig. 2). This precursor is characterised by an accelerating emission rate, leading in two very strong EMA with the following characteristics: The first and the second anomaly lasted for 12 and $17 \mathrm{~h}$, respectively. The cessation interposed between these two signals has duration of almost $12 \mathrm{~h}$. The second anomaly ceased at about $9 \mathrm{~h}$ before the EQ. The larger 

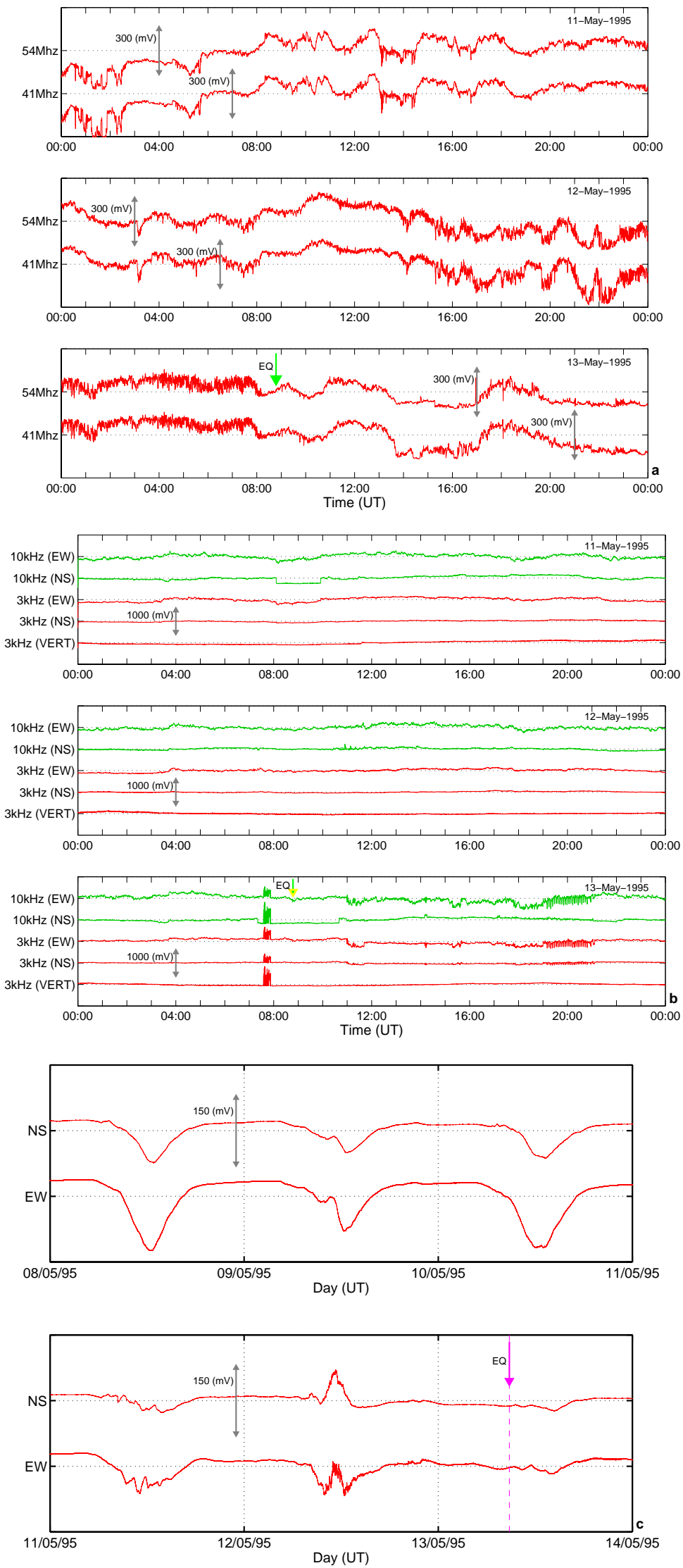

Fig. 1. EMA detected at Zante station prior to the $M_{S}(\mathrm{ATH})=6.6$ Kozani-Grevena EQ on 13 May 1995 at 08:47:13 UT, (a) at the two electric dipoles at 41 and $54 \mathrm{MHz}$, (b) at the 3 and $10 \mathrm{KHz}$ magnetic loop antennas and (c) at the STWA sensors at the DC-ULF band. 

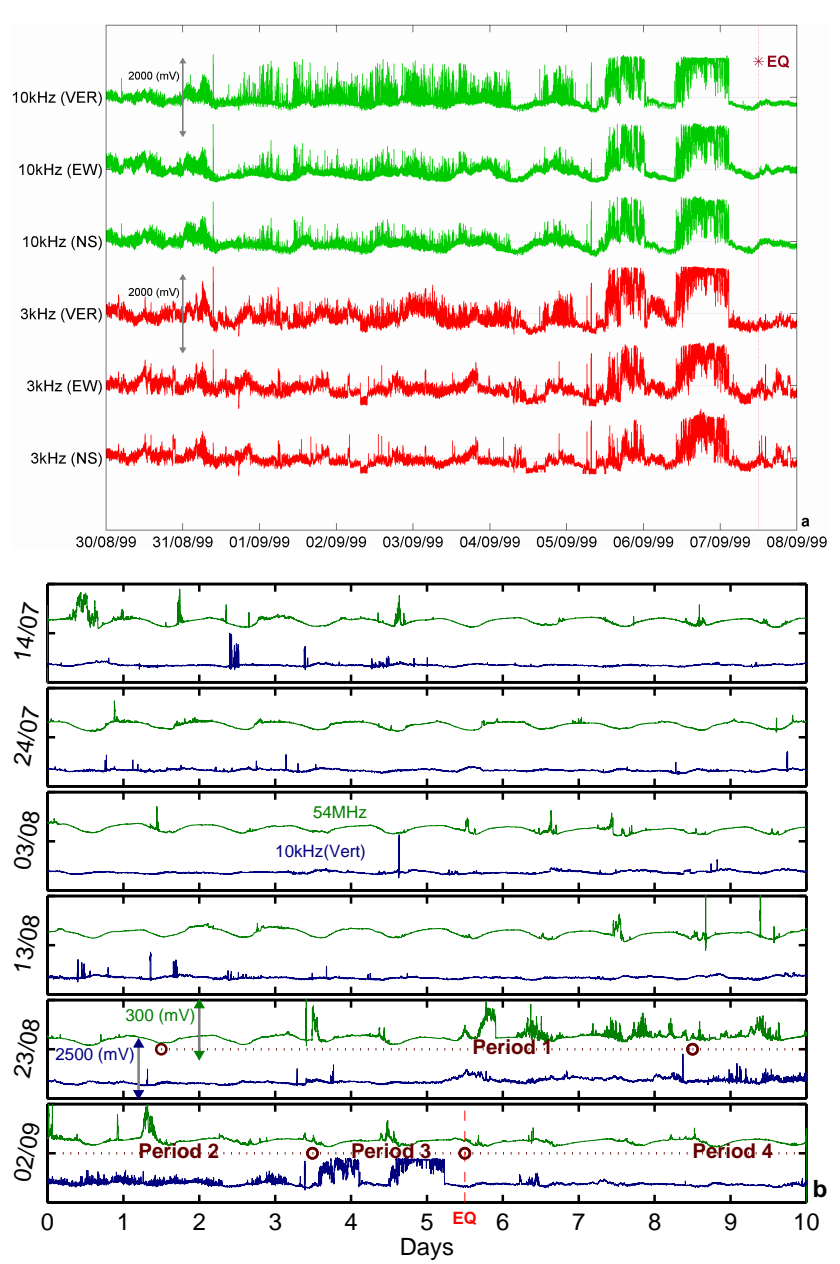

Fig. 2. (a) EMA from Zante station at the 3 and $10 \mathrm{KHz}$ loop antennas which preceded the $M_{S}(\mathrm{ATH})=5.9$ Athens EQ on 7 September 1999 at 11:56:50 UT, from 30 August to 8 September 1999 (sampling rate 1 sample $/ \mathrm{sec}$ ). (b) Records from Zante station at $10 \mathrm{kHz}$ (blue line) and $54 \mathrm{MHz}$ (green line) for the time period from 14 July 1999 to 12 September 1999 (1 min averaged data). The red dashed line denotes the exact time of the EQ occurrence.

anomaly, the second one, contains approximately $80 \%$ of the total electromagnetic energy received. It is remarkable that no EMA were detected in the VHF band as opposed to other cases: e.g. as in the precursory signals of the K-G EQ. With regard to the absence of VHF signals, we note that the upper part of the crust did not rupture during the Athens EQ (Kontoes et al., 2000). Notice that the eye perceives bursts in the VHF time-series (Fig. 2). However, there are no arguments that could relate these bursts with a micro-fracturing process in the Athens earthquake focal area (see comment in Sect. 10).

\section{Fault model signatures in electromagnetic precursors}

The presence/absence of surface ruptures explains the presence/absence of electromagnetic detection in the VHF band. The following finding further supports the concept that the features of the pre-seismic EMA are possibly correlated with the fault model characteristics suggested for each EQ: The fault modelling of the Athens EQ, based on information obtained by radar interferometry (Kontoes et al., 2000), predicts two faults. The main fault segment is responsible for $80 \%$ of the total energy released, with the secondary fault segment for the remaining $20 \%$. Moreover, a recent seismic data analysis carried out by M. Kikuchi indicates that a twoevent solution for the Athens EQ is more likely than a single event solution (Eftaxias et al., 2001b).

Based on this surprising correlation in the energy domain between the two strong pre-seismic kHz EM signals and two faults of the Athens EQ, the idea that the detected pre-seismic electromagnetic emissions are probably emitted from the focal area appears reasonable.

\section{Heterogeneity fingerprints in the EMA patterns}

Experimental and theoretical investigations during the last decades have established that the overwhelming number of fracture phenomena in solid systems is strongly influenced or even controlled by heterogeneity. Laboratory tests reveal that the more homogeneous the medium is, the greater the difficulty in identifying the diagnostic features of its fracture, and vice versa (Morgounov, 2001). Hence, the longer the disorder is the stronger, and more useful, the precursors to rupture are (Mogi, 1962; Sornette, 2000); the more frequent the interaction of crack with non-homogeneities is, the longer the fracture route is (Swanson, 1984); the bigger the variance of spatial distribution of local strengths is, the earlier the stage at which fracture precursors appear (Shamina, 1981; Gershenzon et al., 1989). Note that the "democratic fiber bundle model" (DFBM) exhibits an interesting transition as function of the amplitude of the disorder: there exists a tricritical transition, from a Griffith-type abrupt rupture regime to a progressive damage regime as the disorder increases (Sornette et al., 2000). One can see signatures of the above-mentioned arrangements in the patterns of the detected preseismic signals (Figs. 1 and 2). Indeed, on one hand, the geological and tectonic regime-focal area of Athens EQ is significantly more heterogeneous than those of Kozani-Grevena EQ (Eftaxias et al., 2000, 2001a). On the other hand, in the case of Athens EQ, the preseismic VLF electromagnetic emissions exhibit: larger mean amplitudes, significantly longer duration and significant shorter lead-time (Figs. 1 and 2).

In general, the reader, familiar with magnitude records of earthquakes or of acoustic/electromagnetic records from laboratory experiments, would recognize in the broadly distributed intermittency of our data associated with the Athens EQ (see Fig. 2) the pattern of rupture of heterogeneous system including two strong asperities. Note the temporal clustering of breaking events and the large time intervals of quiescence; the breaking process is very discontinuous in time. During such time intervals of quiescence all cracks are stressed below their cohesion thresholds. The bursts occur relatively often for small times and become more rare later 
(Tzschichholz and Herrmann, 1995). "As the global rupture is approached, there are fewer and fewer bursts since they are larger and larger" (Sornette, 2000).

The importance of heterogeneity to control the nature of rupture processes seems a ubiquitous property (Sornette, 2000). We identify signatures of the order of heterogeneity of each focal area in the patterns of the associated preseismic signals. This evidence further supports the idea that the EMA are probably emitted from the focal area during microfracturing processes.

\section{Seismogenic origin of the detected EMA in terms of laboratory experiments}

The physics of seismicity structure is not yet fully understood. It is practically impossible to directly monitor the states of stress and strain in the lithosphere. One solution to this problem is to model the seismicity in the laboratory by means of acoustic and electromagnetic emission. It is a tricky task to extrapolate patterns of physical processes observed in the laboratory to the field, however, these results should not be ignored. In fact, various experiments on rock samples revealed that $\mathrm{AE}$ emissions exhibit surprising similarities to earthquakes in their behaviour: They obey the wellknown Gutenberg-Richter frequency magnitude relation for earthquakes; their fault plane solutions are similar in type to those of earthquakes; the aftershock AE activity shows the same statistical regularities as natural aftershocks, following the Omori law; the AE emissions have a spatial fractal structure typical of seismicity (Ponomarev et al., 1997 and references therein). In parallel, many researchers have reported simultaneous acoustic and electromagnetic emissions in rock samples under increasing stress. This fact implies that electromagnetic emission is truly closely connected to the micro-cracking process in the sample (Yamada et al., 1989; Fifolt et al., 1993; Petrenco and Gluschenkov, 1996; Mavromatou and Hadjicontis, 2001). Notice that field observations in cave confirmed the simultaneous acoustic and electromagnetic emissions under real conditions (Bella et al., 1994). The offspring of these findings motivated us to investigate a possible correspondence in the behaviour of the frequency content between the field detected EMA and the laboratory observed acoustic and electromagnetic emissions in rock samples, during different deformation stages (Eftaxias et al., 2002).

Onhaka and Mogi (1982) carried out lab experiments on acoustic emissions in rocks at four different narrow frequency bands including a low frequency window $l$ (with sensitivity peaks around $30 \mathrm{kHz}$ and a high frequency window $h$ (with sensitivity peak around $1 \mathrm{MHz}$ ). They found changes in the frequency content of AE activity during five progressive deformation stages. During the third stage (III) (with a stress range from $21-98 \%$ of the failure strength) the acoustic activity begins to build up and increases exponentially when the loading rate is constant, while, the acoustic emission rate $n(l)$, at low frequency window $(\mathrm{kHz})$ is systematically smaller than the acoustic emission rate $n(h)$ at higher frequency window $(\mathrm{MHz})$. At the fourth stage (IV), which is the pre-failure stage (90-100\% of the failure strength), the increase rate of $n(l)$ becomes more rapid than that of $n(h)$ which means that this stage is characterised by a higher level of

$$
\frac{n(l)}{n(l)+n(h)} \text {. }
$$

Notice that a similar shift of the frequency content of the electromagnetic emission to lower frequencies was also observed in laboratory experiments of rupture (MavromatouHadjiconti, 1995). This is a reasonable behaviour if we accept that cracks interact and coalesce to form larger cracks, as the failure approaches, and larger source dimensions are associated with the increase in the low frequency content of both acoustic and electromagnetic signals (Meredith, 1990; Meredith et al., 1990).

The pre-seismic sequence of EMA before K-G earthquake was first recorded at the high frequency $(41 \mathrm{MHz}$ and $54 \mathrm{MHz}$ ) band. Then, as the preparation process of the impending earthquake was developing with time in the Earth, and just before rock failure $\sim(90-60 \mathrm{~min})$, very strong electromagnetic signals appeared in the low frequency $(3 \mathrm{kHz}$ and $10 \mathrm{kHz}$ ) bands. This behaviour is in agreement with the above report of Ohnaka and Mogi.

The evaluation of the preseismic electromagnetic anomalies in terms of laboratory experiments further supports the concept that the EMA are probably emitted from the focal area during microfracturing processes. Moreover, if we consider that the preparation process of a large EQ and that of local fracturing of a rock specimen have a similar physical basis, then, the observed frequency shift from $\mathrm{MHz}$ to $\mathrm{kHz}$ might possibly be related to the last stage of the EQ preparation process in the focal area. The field experience encourages this concept. Thus, the extended Chinese field experience for this type of EMA, based on a large number of observations, shows that the pre-seismic anomalies are detected earlier in the VHF than in the VLF frequency bands (Qian et al., 1994). Moreover, many workers have reported that the number of VLF pulse-like signals increase clearly just before the EQs (Fujinawa et al., 1997 and references therein).

\section{EMA as the by-product of the foreshock activity}

Many authors suggest that the pre-seismic electromagnetic activities could be considered as a by-product of the foreshock activity in the electromagnetic domain (Uyeda, 1996; Kanamori, 1996; Molchanov, 1999; Hayakawa et al., 1999, 2000). Recently, Telesca et al. (2001) and Balasco et al. (2002), based on a new fractal approach, have found an observational evidence of correlations between the time variation of parameters describing the temporal fluctuations of geo-electromagnetic signals and earthquakes that occurred in the area investigated. Thus, we attempt to test the hypothesis if the understudy EMA could be considered as the by-product of the foreshock activity. 
The Athens case: The Athens, Greece, earthquake of 7 September 1999 occurred in a region of low seismicity at a distance $\sim 600 \mathrm{~km}$ from the epicentre of, and only 21 days after, the large $\left(M_{w}=7.5\right)$ Izmit, Marmara sea, earthquake of 17 August 1999. Brodsky et al. (2000) have shown that the Izmit earthquake triggered widespread regional seismicity in Greece. They speculate "dynamic triggering on regionalscale results in countrywide episodes of increased seismicity or superwarms". On the other hand, the precursory electromagnetic anomaly, which is characterised by an accelerating emission rate, has been detected from 31 August to 7 September 1999, i.e. within the time window of the triggered seismicity in Greece from Izmit EQ. If we keep in mind that the preseismic EMA is embedded in a long duration quiescence period concerning the detection of electromagnetic disturbances at the VLF frequency band, the concept that the captured VLF-anomaly is strongly associated with the triggered seismicity can be accepted.

The following finding worth attention: a recent time-tofailure analysis (Papadopoulos, 2002) showed that the process of small EQs generation, at a distance of about one source dimension $(\sim 30 \mathrm{~km})$ from the Athens EQ epicentre, started accelerating very slowly from the beginning of 1994. However, only immediately after the Izmit EQ the process culminated with short-term foreshock activity. The author concludes, "We get a clear short-term acceleration similar to the classic foreshock seismicity increase". Notice that the main shock was preceded by four measurable immediate foreshocks of $M_{L} 3.2,2.5,2.5$ and 3.2 within $18 \mathrm{~min}$ of the main shock (Papadopoulos, 2002). This finding strongly supports the concept that the observed EMA could be considered as the by-product of the foreshock activity associated with the Athens EQ.

The Kozani-Grevena case: The K-G earthquake was preceded within $30 \mathrm{~min}$ of the main shock by five foreshocks with $M>3.5$ clustered within $2 \mathrm{~km}$ of one another about 5 to $10 \mathrm{~km}$ from the main shock epicentre (Bernard et al., 1997). Thus, the unusual foreshock activity for the aseismic $\mathrm{K}-\mathrm{G}$ region was reported a few minutes after the detection of the $\mathrm{kHz}$ EMA and a few minutes before the EQ. This size of foreshock clustering correctly fits the correlation law with the main-shock magnitude obtained by (Dodge et al., 1995) for Californian earthquakes (Bernard et al., 1997). Hence, the observed foreshocks may be possibly regarded as a fingerprint of a local dynamic rupture of asperities in the weak zone associated with the nucleation on the main rupture.

Consequently, the idea that the under study preseismic electromagnetic activities could imply an underlying structural instability, that belongs to the final stage of the EQ preparation process within the focal area, can be accepted.

\section{Possible EMA-Nucleation Phase correlations}

Observation of foreshocks is the most convincing evidence that there is a nucleation stage before major EQs. So, the offspring of the above mentioned data motivated us to inves- tigate the presence of signatures of the underlying nucleation phase of EQ in the characteristics of EMA. Many hypotheses have been presented concerning the nucleation and growth of faults in brittle rocks. Most of these views are based on experiments that use acoustic emission events to monitor faulting processes. The abrupt increase in the AE activity, which is characterised by an accelerating emission rate, is considered as the most commonly effective way to recognise the nucleation stage (e.g. Kranz and Scholz, 1977; Lockner and Madden, 1991; Lockner, 1993; Ohnaka, 2000 and references therein; Morgounov, 2001). Wu and Thomsen (1975), Lockner and Byerlee (1980), Ohnaka (1983) define the onset of the tertiary creep by a law close to exponential rise of acoustic emission.

If we consider that the preparation process of a large EQ and that of local fracturing of rock specimen have a similar physical basis, then, an accelerating emission rate is highly to be considered as a precursory electromagnetic signature of the nucleation phase.

Indeed, we observe the presence of accelerating activity in the detected anomalies (Figs. 1 and 2). An interesting outcome of the wavelet spectral analysis is the observation that both the K-G and Athens EQs were preceded by an accelerating electromagnetic emission (Eftaxias et al., 2001b, 2002). We remind the surprising correlation found in the energy domain between the two strong preseismic $\mathrm{kHz}$ EM signals and the two faults of Athens earthquake (Eftaxias et al., 2001). This result further supports the concept that the detected EMA were correlated with the nucleation phase of the impending $\mathrm{EQ}$.

It is also worth to mention that Ohnaka (2000) states: "for large earthquakes, the time required the rupture to grow from a slow phase of the rupture velocity to the phase of high speed, is of the order of a few to a few tens of hours". Indeed, the precursory VLF-VHF EMA appeared in this time window.

\section{Electromagnetic evidence of the underlying preseis- mic critical stage}

There is accumulating evidence through computer simulations, theoretical approaches and experimental findings that large earthquake exhibits behaviour similar to critical phenomena. If we assume a model where any measure $\varepsilon(t)$ of seismic release at time $t$, close to the time of a large earthquake $t_{c}$, obeys a power law (Bufe and Varnes, 1993; Chelidze, 1982)

$\varepsilon(t)=A+B \cdot\left(t_{c}-t\right)^{m}$

(where, $A$ and $B$ constants, and $m$ a critical exponent), then, one may guess that the associated precursors may follow power laws, which characterize the critical phenomena. This idea has already been exploited in a seismological sense for predicting large earthquakes (Sornette, 2000). The observation of an accelerating electromagnetic activity prior to K-G 

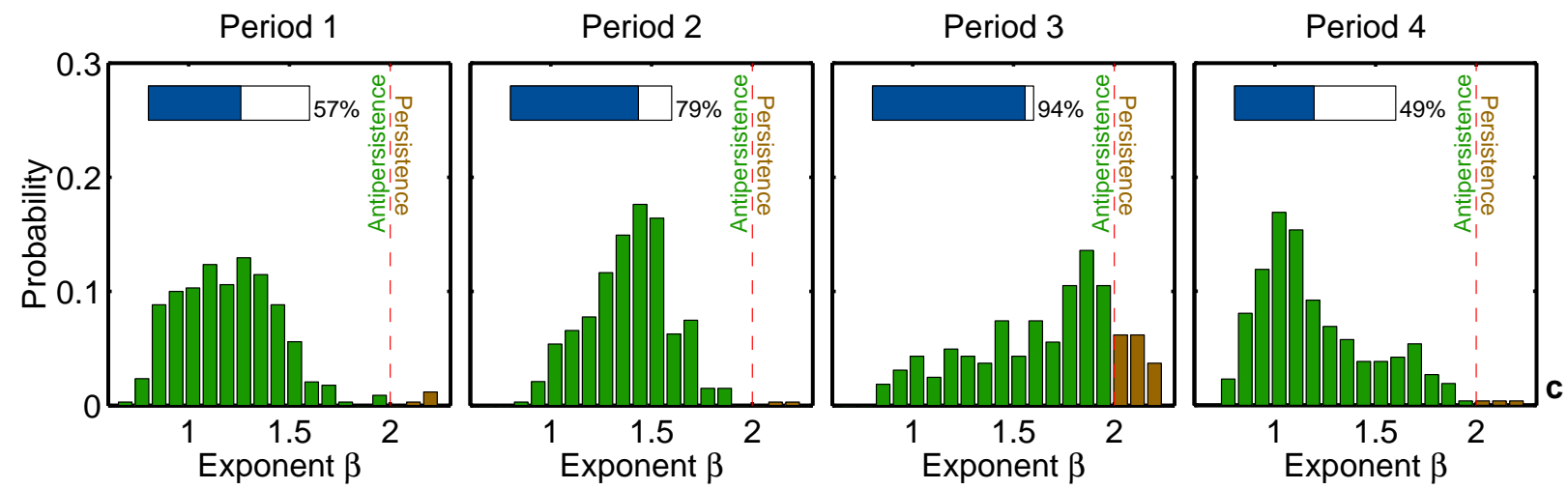

Fig. 3. The probability distribution of the spectral exponent $\beta$ corresponding to segments with linear coefficient $r>0.85$ for four time slices as presented in Fig. 2b. The bars on the top show the percentage of segments with $r>0.85$. It is clear that as we approach the time of the earthquake, cases with $r>0.85$ increases to $94 \%$ (period 3) whereas after the EQ this value drops again. In each panel the dashed line at $\beta=2$ separate the regions of antipersistence/persistence behaviour of the electromagnetic time-series.

and Athens EQs (Eftaxias et al., 2001b, 2002) could have indirectly implied that the system had approached criticality. Then, the possibility that the evolution of the Earth's crust toward critical state takes place not only in a seismological but also in an electromagnetic sense before an earthquake is plausible.

We have treated the statistical properties of earthquake occurrence as a kind of critical phenomenon in view of electromagnetic precursors (Kapiris et al., 2002; Contoyiannis et al., 2002). Thus, we have performed the statistical analysis of the electromagnetic time series in terms of power spectral density. In particular, if the underlying mechanism of the detected EMA was a critical one, then a power-law spectrum of the emission is expected

$S(f)=\alpha \cdot f^{-\beta}$.

In a $\log S(f)-\log f$ representation the power spectrum is a straight line with $\beta$ constituting its linear spectral slope. The spectral amplification $\alpha$ quantifies the power of the spectral components following the power spectral density law. We calculated the parameters $\beta, \alpha$ and $r$, where $r$ is the linear correlation coefficient of the power law fit, dividing the signal into successive segments of 1024 samples, in order to study their time evolution (sampling rate $1 \mathrm{~Hz}$ ).

Characteristically, the analysis of the $10 \mathrm{kHz}$ EMA associated with the Athens EQ reveals the presence of a power law (1) indicating both a fractal geometry of the underlying dynamical system as well as long-range correlations (Kapiris et al., 2002). The time variation of the dynamical parameter $\beta$ (that indicates the strength of the signal's irregularity) is shown in Fig. 3. We recognize a systematical increase of the exponent $\beta$ approaching the $\mathrm{EQ}$. The $\beta$-values are maximum, $\sim 1.8$, in the two strong bursts. This is a reasonable behaviour if we accept that cracks interact and coalesce to form larger cracks, as the failure approaches, and larger source dimensions are associated with the increase in the low frequency content of both acoustic and electromagnetic signals. Notice that this behaviour fails just after the EQ. Note that as we approach the time of the earthquake segments with $r>0.85$ increases to $94 \%$ (period 3).

It should be noted that the analysis of the VLF anomaly $(3 \mathrm{kHz})$ associated with the $\mathrm{K}-\mathrm{G}$ earthquake also reveals the presence of a power law with $r=0.92$ and $\beta=1.93 \pm 0.03$. The spectral exponent $\beta$ is related to another exponent, the Hurst exponent $H$, by the formula (Turcotte, 1992).

$\beta=2 H+1$.

$H$ lies anywhere in the range $0<H<1$ and characterises the persistence properties of the electromagnetic time series according to the following scheme: (i) for $H=0.5$ (i.e. $\beta=2$ ) there is no correlation between the process increments and we are in a standard diffuse regime. As is well known, in the random walk which models classical Brownian diffusion the walker follows a random path driven by memoryless dynamics; (ii) the range $0.5<H<1(2<\beta<3)$ suggests persistence of the signal (super-diffusion), i.e. if the electromagnetic fluctuations increase in one time interval, it is likely to continue increasing in the period immediately following. The accumulation of fluctuations is faster than in classical Brownian motion; (iii) the range $0<H<0.5$ $(1<\beta<2)$, on the contrary, suggests anti-persistence or clustering (sub-diffusion), i.e., if the fluctuations increase in one period, it is likely to continue decreasing in the period immediately following, and vice versa; (iv) at the limit $H=0(\beta=1)$ it does not grow at all and the signal is stationary. Concerning our data, as the EQ is approaching, the "mean" exponent $H$ gradually increases (see Fig. 3). Inside the two strong bursts (i.e. period 3 in Fig. 3 ) the exponent $H$ rise above the value of 0.5 . It is remarkable that the theoretical study carried out by Tzschichholz and Herrmann (1995) suggests that events inside a strong burst seem uncorrelated. This behaviour of $H$ indicates that the electromagnetic fluctuations become less anti-correlated or correlated approaching the main event, as the dominant exponents $H$ are closer to 0.5 . This temporal evolution of the dynamical parameters 


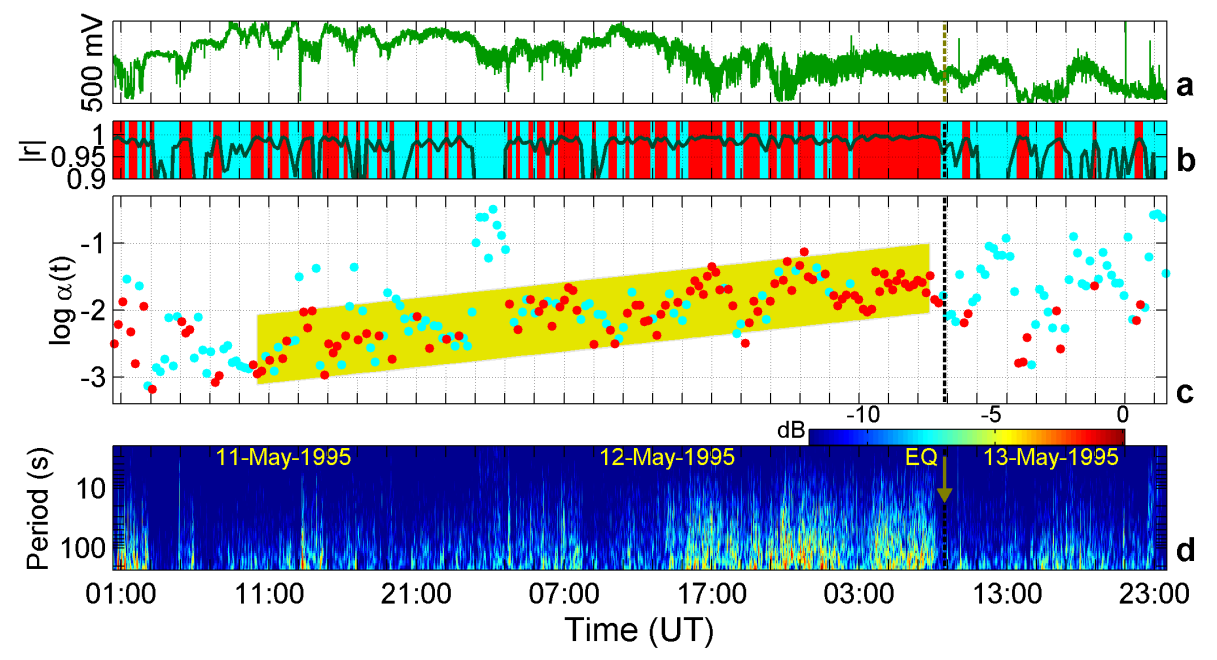

Fig. 4. (a) The $41 \mathrm{MHz}$ electromagnetic anomaly recorded prior to the 13 May 1995 Kozani-Grevena earthquake (the event is indicated with a vertical dashed line in all panels); (b) the variation of the linear coefficient $r$ of the power law spectral fitting (solid line). Red shaded zones correspond to values $r>0.98$; (c) time-dependence of the spectral amplification parameter $\alpha$. The shaded zone corresponds to the interval of exponential increase of $\alpha$; and (d) the wavelet power spectral amplitude evolution of the electromagnetic anomaly. The intensity scale increases from blue to red.

$\beta, H$ seems to be compatible with the last phase of the $\mathrm{EQ}$ generation.

We draw the attention to the following experimental fact: An increase of exponent $\beta$ was observed in the VHF preseismic EM time-series during the last few tens of hours prior to the Kozani-Grevena EQ (Kapiris et al., 2002). These results confirm the Italian experience described in Cuomo et al. (1998), Telesca et al. (2001), and Balasco et al. (2002). Moreover, an increase of exponents $\beta$ has been observed in the tail of the electromagnetic ULF-time series associated with (i) the Biak EQ $\left(M_{w}=8.2\right)$, on 17 February (see Fig. 4 in Hayakawa et al., 2000), and the Guam EQ $\left(M_{s}=8.0\right)$ on 8 August 1993, see Fig. 2 in Hayakawa et al., 1999).

Summing up, the increase of $\beta$ (and $H$ ) approaching the EQ seems to have a universal character. Thus, the concept that this increase is a candidate short-term pre-seismic signature is reasonable.

\section{Nucleation signatures in the EM power spectrum}

We remind that an abrupt increase in the AE activity, which is characterised by an accelerating emission rate, is considered as the most commonly effective way to recognise the nucleation stage. Spectral analysis permits a quantitative approach concerning the presence of an accelerating emission stage in the electromagnetic precursors (Fig. 4). The temporal evolution of $r$ (Fig. 4b) reveals a progressive increase of the time intervals with excellent $r$, i.e. $r>0.98$, as approaching the K-G EQ (Fig. 4a). This behaviour may constitute signature of an underlying precursory critical mechanism. We focus on the spectral amplification $\alpha$ that measures the "instant" intensity of the electromagnetic source. The time evolution of the estimated $\alpha$ corresponding to segments with $r>0.98$ is shown in Fig. 4c. We observe that the temporal evolution of $\alpha$, exhibits a nearly exponential increase, during the last few tens of hours prior to the K-G EQ. It is reminiscent that in the laboratory experiments of rupture several studies have observed an exponential acceleration of the energy release before the macroscopic rupture. Note that is often difficult to distinguish in real data an exponential increase from a powerlaw increase.

This increase in the signal's intensity approaching the event can be also qualitatively seen in the wavelet scalogram (Fig. 4d), which permits quantitative monitoring of the evolution of the transient signals, by decomposing a time-series to linear superposition of identical predefined mathematical waveforms (wavelets), each with finite duration and narrow frequency content. We have chosen the wavelet analysis based on the Morlet mother wavelet. Figure $4 \mathrm{~d}$ also shows a progressive increase of the $41 \mathrm{MHz}$ event-rate with time, from 11 to 13 May 1999.

These results further strengthen the hypothesis that the observed electromagnetic anomalies are correlated with the underlying nucleation phase of the K-G EQ.

The presence of a power law in the electromagnetic activity and its capture with an accelerating rate seems to constitute basic characteristics of the VLF-VHF precursory signals. These signatures do not exist in the observed $54 \mathrm{MHz}$ bursts preceding the Athens earthquake (see Fig. 2). Notice that the vast majority of these bursts appear during daytime. This evidence does not support the seismogenic origin of these bursts. In general there are no arguments, based on our knowledge, that could relate these bursts with a microfracturing process in the focal area of the Athens earthquake. 


\section{A challenging prospective concerning the epicentre location}

The epicentre location before the EQ is very important. Main and Meredith (1990) suggest: "The critical stage for useful earthquake warning would be to reliably detect the transition from energy release predominantly in the volume surrounding the focal area to that on the fault itself". Remind that at a distance of about one source dimension $(\sim 30 \mathrm{~km})$ from the Athens EQ epicentre, we get a clear short-term acceleration similar to the classic foreshock seismicity increase. This seismicity included four measurable immediate foreshocks of $M_{L}$ 3.2, 2.5, 2.5 and 3.2 within 18 min of the main shock (Papadopoulos, 2002). Moreover, the K-G EQ was also preceded by five foreshocks clustered within $2 \mathrm{~km}$ of one another about 5 to $10 \mathrm{~km}$ from the main shock epicentre. The detection of a sequence of electromagnetic precursors might support the identification of shocks as foreshocks. After the detection of the preseismic electromagnetic activity, if we observe a "clear short-term acceleration similar to the classic foreshock seismicity increase", the associated fault might be a candidate source of the major EQ. Consequently, a proper combination of the information inferred from the various precursory anomalies can lead to the estimation of the epicentre of the impending EQ. We remind that the ULF EMA have longer lead-time than the VLF-VHF EMA. An appropriate combination of ULF-geoelectric potential changes (SES) physical properties can lead to the estimation of the epicentre of the impending EQ (VAN-method) (Varotsos, et al., 1996). Recently, Du et al. (2002) have presented a new polarization technique, which can be used to locate the epicentre through triangulation by analysing the ULF electromagnetic emissions after removing the background disturbances at each station. A challenging prospective could be the possibility to estimate the location of the forthcoming earthquake from independent precursory data sets, detected at different time periods, i.e. from such as the DC-ULF and VLF-VHF signals. The achievement of converging estimations would definitely improve the chances of prediction.

\section{ULF EMA detected on the STWA sensors}

Concerning the ULF signals detected some hours before the K-G EQ on the recordings of the EW/NS STWA sensors (Fig. 1c), they are out of the frequency range we deal with in this paper. However, it is worthy to make some comments. There was a few hours anomalous increase to a remarkably high level of activity with higher frequency components, starting approximately $20 \mathrm{~h}$ before the earthquake causing a clear inversion of the usual daily variations (Fig. 1c). Notice that a similar anomaly was detected before the Egion-Eratini earthquake (Eftaxias et al., 2000, 2001a). Unfortunately, the equipment of the STWA sensors was not operating at the time of Athens earthquake. A clear theoretical interpretation of these precursory anomalies is still lacking and it will be the subject of a further research.

\section{A possible propagation mechanism of VLF EMA}

There is no consensus on the mechanism of the long distance propagation of EMA. Concerning the VLF EMA, both the K-G and the Athens EQs were very shallow. Thus, the earthquake may have produced any of the surface electrical affects, or other changes, which presumably are required to launch VLF signals into the atmosphere. For example, the following scenario cannot be excluded: the preseismic processes in the focal zone involved a surface zone approximately of the same size. Several authors in connection to laboratory experiments have discussed electrification by microfracturing, i.e. the appearance of spontaneous charge production associated with the opening of microcracks. Such emissions of charges from stressed rocks around the focal area accompanying micro fracturing can act as a current system in a conductor with a length of the order of the subsequent fracture generated by the earthquake (Biagi, 1999). In general, the radiation pattern of an antenna can be effectively excited, only by certain frequencies corresponding to the characteristic length scales of the antenna, e.g. $\mathrm{kL} \sim 1$. For both cases (K-G and Athens EQs), the spatial scale L is of the order of $10 \mathrm{~km}$ (Meyer et al., 1998; Kontoes et al., 2000). Therefore, the frequency of the radiated signal in the free space is of the order of a few $\mathrm{kHz}$. This finding support the concept that the detected $3 \mathrm{kHz}$ and $10 \mathrm{kHz}$ prior to the three destructive EQs in Greece are produced by a direct effect of emission at a few $\mathrm{kHz}$ on so, caused by surface zone of the forthcoming EQ that act as an antenna. On the other hand, our experience suggests that the presence/absence of VHF signal in the preseismic sequence of electromagnetic anomalies depends on the presence/absence of surface ruptures. This fact supports the hypothesis that the reported VHF precursory anomalies were also produced during surface microfracturing processes.

\section{The electromagnetic quiescence just before the earthquake}

An interesting characteristic of the EMA detected prior to all three above mentioned destructive EQs in Greece is the quiescence period at all frequency bands just before the EQ (Eftaxias et al., 2000, 2001a). This finding confirms the international experience. Indeed, a general observation from all of the experiments designed to detect precursors is that signals are observed before the EQs, but rarely are signals observed at the time of EQs (Morgounov, 2001 and references therein).

The phenomenon of acoustic/electromagnetic quiescence close to the peak stress has been reported to occur close to the final failure in laboratory studies on rocks (Meredith et al., 1990; Morgounov, 2001). In particular Morgounov (2001) concludes that the test in the condition of self-regulated stress relaxation indicates that electromagnetic emission precedes the phase of macroscopic failure and is absent during the generation of macro cracks, which, on the whole, correspond to 
the absence of electromagnetic eruption at the onset of the earthquake.

Yamada et al. (1989) proposed a possible mechanism for EM emission that also explains the EM gap before the failure. According to their suggestion, EM emission in a rock is due to electrification of a fresh surface. EQs are generally considered to be a result of shear faulting that connects pre-existing small cracks. They state, "It is reasonable to expect anomalous EM emissions associated with small tensile cracks before an earthquake but not at the main shock (which is mainly due to a shear crack) which may not be efficient for creation of a fresh surface".

Recently, Matsumoto et al. (1998), using an electromagnetic model of a geological fault (Ikeya et al., 1997) have also explains the VHF quiescence before the failure.

However, much more work is needed to improve the observation and refine the model of precursor electromagnetic gap. We remind that our experience shows that the duration of the electromagnetic quiescence is related with the heterogeneity of the associated focal area: the larger the heterogeneity is, the larger the duration of gap is. This arrangement supports the seismogenic origin of the electromagnetic gap.

\section{The electromagnetic quiescence during the after- shock period}

Our field experience did not find precursory EMA for aftershock sequences (Eftaxias et al., 2000). A possible explanation for the lack of EM perturbations during the aftershock period, based on laboratory experiments of rupture, is that rocks show extreme nonlinear elastic behaviour, hysterisis and discrete memory. If the sample is loaded and unloaded before fracture and loaded again, only a small number of micro-fractures are detected before attaining the previous load (Kaiser effect) (Meredith, 1990). Thus, laboratory experimental results can justify the absence of EMA before an aftershock. The explanation is that the stress required for an aftershock to occur does not exceed the stress needed for the main shock.

\section{Conclusions}

Electromagnetic anomalies from ULF, VLF up to VHF have been observed before recent destructive earthquakes in continental Greece. The features of these signals are possibly correlated with the fault model characteristics of the associated earthquake and with the degree of geotectonic heterogeneity within the focal zone. Thus, the idea that these anomalies are probably emitted from the focal area is reasonable. The time evolution of these electromagnetic sequences reveals striking similarities to that observed in laboratory acoustic and electromagnetic emissions during different stages of failure preparation process in rocks. Thus, the concept that the detected EMA are probably emitted during micro fracturing processes can be accepted. If we consider that the same dynamics governs the large-scale earthquakes and the micro- scopic scale sample rheological structure, the results of this analysis also suggest that the recorded EMA might reflect the nucleation phase of the associated impending earthquake. We focus on the rise of the statistical view of earthquakes. We found electromagnetic fingerprints of an underlying critical mechanism. This result further supports possible EMAnucleation phase correlations. Finally, we conclude that it is useful to combine ULF and VLF-VHF field measurements in an attempt to enhance the understanding of the physics behind these observations and thus to improve the quality of earthquake prediction.

On the basis of our experience we conclude that the VLFVHF electromagnetic precursors do exist and that the development of suitable observational techniques and analysis methods is a promising research direction for EQ precursors study.

\section{References}

Balasco, M., Lapenna, V., and Telesca, L.: $1 / f^{a}$ Fluctuations in geoelectrical signals observed in a seismic area of southern Italy, Tectonophysics, in press, 2002.

Bella, F., Biagi, P., Caputo, M., Della Monica, G., Ermini, A., Plastino, W., and Sqrigna, V.: Electromagnetic background and preseismic anomalies recorded in the Amare Cave (Central Italy), in: Electromagnetic Phenomena Related to Earthquake Prediction (Eds) Hayakawa, M. and Fujinawa, Y., Terrapub, Tokyo, 181-192, 1994.

Bernard, P., Pinettes, P., Hadjidimitriou, P., Scordilis, E., Veis, G., and Milas, P.: From precursors to prediction: a few recent cases from Greece, Geophys. J. Int., 131, 467-477, 1997.

Biagi, P.: Seismic effects on LF radiowaves, in: Atmospheric and Ionospheric Electromagnetic Phenomena associated with Earthquakes (Ed) Hayakawa, M., Terrapub, Tokyo, 535-542, 1999.

Brodsky, E., Karakostas, V., and Kanamori, H.: A new observation of dynamically triggered regional seismicity: Earthquakes in Greece following the August, 1999 Izmit, Turkey earthquake, Geophys. Res. Lett., 27, 17, 2741-2744, 2000.

Bufe, G. and Varnes, D.: Predictive modelling of the seismic cycle of the greater San Francisco bay region, J. Gepophys. Res., 98, 9871-9883, 1993.

Chelidze, T.: Percolation and fracture, Phys. Earth Planet. Inter., 28, 93-101, 1982.

Clarke, P., Pradissis, D., Briole, P., England, P., Parsons, B., Billiris, H., Veis, G., and Ruegg, J.: Geodetic investigation of the 13 May 1995 Kozani-Grevena (Greece) earthquake, Geophys. Res. Lett., 24, 6, 707-710, 1997.

Contoyiannis, Y., Diakonos, F., Kapiris, P., Peratzakis, A., and Eftaxias, K.: Fingerprints of intermittent and critical behaviour of pending earthquake in electromagnetic anomalies, EGS XXVII General Assembly, NH062, EGS02-A-00244, 2002.

Cuomo, V., Lapenna, V., Macchiato, M., Serio, C., and Telesca, L.: Linear and nonlinear dynamics in electrical precursory time series: implications for earthquake prediction, Tectonophysics, 287, 279-298, 1998.

Dodge, A., Beroza, C., and Ellswroth, L.: Evolution of the 1992 Landers, California, foreshock sequence and its implications for earthquake nucleation, J. Geophys. Res., 100, 9865-9880, 1995. 
Du, A., Huang, Q., and Yang, S.: Epicenter location by abnormal ULF electromagnetic emissions, Geophys, Res. Lett., 29, 10, 641-64-3, 2002.

Eftaxias, K., Kopanas, J., Bogris, N., Kapiris, P., Antonopoulos, G., and Varotsos, P.: Detection of electromagnetic earthquake precursory signals in Greece, Proc. Japan Acad., 76(B), 45-50, 2000.

Eftaxias, K., Kapiris, P., Polygiannakis, Y., Hadjicontis, V., Chelidze, Z., Zilpimiani, D., and Chelidze, T.: Seismogenic radioemission as a signature of the earthquake preparation process, $\mathrm{J}$. of the Georgian Geophysical Society, Issue (A), Physics of Solid Earth, 6(a), 3-16, 2001a.

Eftaxias, K., Kapiris, P., Polygiannakis, J., Bogris, N., Kopanas, J., Antonopoulos, G., Peratzakis, A., and Hadjicontis, V.: Signatures of pending earthquake from electromagnetic anomalies, Geophys. Res. Lett., 28, 3321-3324, 2001b.

Eftaxias, K., Kapiris, P., Dologlou, E., Kopanas, J., Bogris, N., Antonopoulos, G., Peratzakis, A., and Hadjicontis, V.: EM anomalies before the Kozani earthquake: A study of their behavior through laboratory experiments, Geophys. Res. Lett., 29, 8, 69-1-69-4, 2002.

Fifolt, A., Petrenko, F., and Schulson, M.: Preliminary study of electromagnetic emissions from cracks in ice, Phil. Mag., B, 67, 289-299, 1993.

Fraser-Smith, A. C., Bernardi, A., McGill, P. R., Ladd, M. E., Helliwell, R. A., and Villard, O. G.: Low-frequency magnetic field measurements near the epicenter of the $M_{S} 7.1$ Loma Prieta earthquake, Geophys. Res. Lett., 17, 1465-1468, 1990.

Fujinawa, Y., Takahashi, K., Matsumoto, T., and Kawakami, N.: Experiments to Locate Sources of Earthquake-related VLF Electromagnetic Signals, Proc. Japan Acad., 73(B), 33-38, 1997.

Gershenzon, N., Gokhberg, M., Karakin, A., Petviashvili, N., and Rykunov, A.: Modeling the connection between earthquake preparation processes and crustal electromagnetic emission, Physics of Earth and Planetary Interiors, 57, 129-138, 1989.

Gershenzon, N. and Bambakidis, G.: Modelling of seismoelectromagnetic phenomena, Russian Journal of Earth Sciences, 3, 4, 247-275, 2001.

Gokhberg, M., Morgunov, V., and Tomizawa, I.: Experimental measurements of electromagnetic emissions possibly related to earthquake in Japan, J. Geophys. Res., 87(B9), 7824-7828, 1982.

Gokhberg, M., Morgunov, V., and Pokhotelov, O.: Earthquake Prediction, Seismo-Electromagnetic Phenomena, Gordon and Breach Publishers, Amsterdam, 193 pp., 1995.

Hadjicontis, V. and Mavromatou, C.: Laboratory Investigation of the Electric Signals Preceding earthquakes, in: A Critical Review of VAN, (Ed) Lighthill, Sir J., World Scientific Singapore, 105$117,1996$.

Hayakawa, M. and Fujinawa, Y. (Eds): Electromagnetic Phenomena Related to Earthquake Prediction, Terrapub, Tokyo, 677 pp., 1994

Hayakawa, M., Fujinawa, Y., Evison, F. F., Shapiro, V. A., Varotsos, P., Fraser-Smith, A. C., Molchanov, O. A., Pokhotelov, O. A., Enomoto, Y., and Schloessin, H. H.: What is the future direction of investigation on electromagnetic phenomena related to earthquake prediction?, in: Electromagnetic phenomena related to earthquake prediction, (Eds) Hayakawa, M. and Fujinawa, Y., Terrapub, Tokyo, 667-677, 1994.

Hayakawa, M. (Ed): Atmospheric and Ionospheric Electromagnetic Phenomena Associated with Earthquakes, Terrapub, Tokyo, 996 pp., 1999.
Hayakawa, M., Ito, T., and Smirnova, N.: Fractal analysis of ULF geomagnetic data associated with the Guam eartquake on $8 \mathrm{Au}-$ gust 1993, Geophys, Res. Lett., 26, 2797-2800, 1999.

Hayakawa, M., Itoh, T., Hattori, K., and Yumoto, K.: ULF electromagnetic precursors for an earthquake at Biak, Indonesia oin 17 February 1996, Geophys, Res. Lett., 27, 10, 1531-1534, 2000.

Hayakawa, M. and Molchanov, O. (Eds): Seismo Electromagnetics Lithosphere-Atmosphere-Ionosphere coupling, Terrapub, Tokyo, 477 pp., 2002.

Ikeya, M., Kinoshita, H., and Matsumoto, H.: A model experiment of electromagnetic wave propagation over long distances using waveguide terminology, JPN. J. App. Phys., 36, 1558$1561,1997$.

Kanamori, H.: A seismologist's view of VAN, in: A critical review of VAN. Earthquake Prediction from Seismic Electric Signals, (Ed) Lighthill, Sir J., World Scientific Publishing Co., Singapore, 339-346, 1996.

Kapiris, P., Polygiannakis, J., Antonopoulos, G., Kopanas, J., Peratzakis, A., and Eftaxias, K.: An observational test of the critical earthquake concept: The electromagnetic point of view, EGS XXVII General Assembly, NP9.05, EGS02-A-00554, 2002.

Kontoes, C., Elias, P., Sykioti, O., Briole, P., Remy, D., Sachpazi, M., Veis, G., and Kotsis, I.: Displacement field and fault model for the September 7, 1999 Athens earthquake inferred from ERS2 satellite radar interferometry, Geophys. Res. Lett. 27, 24, 3989-3992, 2000.

Kranz, R. and Scholz, C.: Critical dilatant volume of rocks at the onset of tertiary creep, J. Geophys. Res., 82, 4893-4898, 1977.

Kusunose, K. and Nishizawa, O.: AE gap prior to local fracture of rock under uniaxial compression, J. Phys. Earth, 34, Suppl., 45-56, 1986

Lighthill, S. (Ed): A critical Review of VAN. Earthquake Prediction from Seismic Electric Signals, World Scientific Publishing Co., Singapore, 276 pp., 1996.

Lockner, D. and Madden, T.: A multiple-crack model of brittle fracture 2. Time-dependent simulations, J. Geophys. Res., 96, 19643-19654, 1991.

Lockner, D.: The role of acoustic emission in the study of rock fracture, Int. J. Rock Mech. Min. Sci., Geomech. Abstr., 30, 883899, 1993.

Main, I. and Meredith, P.: Classification of earthquake precursors from a fracture mechanics model, Tectonophysics, 167, 273283, 1989.

Matsumoto, H., Ikeya, M., and Yamanaka, C.: Analysis of barberpole color and speckle noises recorded 6 and half hours before the Kobe earthquake, Jpn. J. App. Phys., 37, 1409-1411, 1998.

Mavromatou-Hadjiconti, C.: Laboratory Investigation of the Electric Signals Preceding the Fracture of Crystalline Materials, $\mathrm{Ph}$ D Thesis, University of Athens, 1995.

Mavromatou, C. and Hadjicontis, V.: Laboratory Investigation of the Electric Signals Preceding the Fracture of Crystalline Insulators in Earthquake thermodynamics and phase transformations in the earth's interior, (Eds) Teisseyre, R. and Majewski, E., Academic Press, 501-517, 2001.

Meredith, P., Main, I., and Jones, C.: Temporal variations in seismicity during quasi-static and dynamic rock failure, Tectonophysics, 175, 249-268, 1990.

Meredith, P.: Fracture and failure of brittle polycrystals: an overview, in: Deformation Processes in Minerals, Ceramics and Rocks, (Eds) Barder, D. and Meredith, P., Unwin Hyman, London, 5-41, 1990.

Meyer, B., Armijo, R., Massonet, D., De Chabalier, J. B., Delacourt 
C., Ruegg, J. C., Achache, J., and Papanastassiou, D.: Results from combining tectonic observations and SAR interferometry for the 1995 Grevena earthquake: A summary, J. Geodynamics, 26, 2-4, 255-259, 1998.

Mogi, K.: Magnitude-frequency relation for elastic shocks accompanying fractures of various materials and some related problems earthquakes, Bull. Earthquake Res. Inst. Tokyo Univ., 40, 831853, 1962.

Molchanov, O.: Fracturing as an underlying mechanism of seismoelectric signals, in: Atmospheric and Ionospheric Electromagnetic Phenomena Associated with Earthquakes, (Eds) Hayakawa, M. and Fujinawa, Y., Terrapub, Tokyo, 349-356, 1999.

Morgounov, V.: Predictability of earthquakes: State of the Art, Journal Izvestiya Russian Ac. Sci., Fizika Zemli, 35, 1, 72-83, 1999.

Morgounov, V.: Relaxation creep model of impending earthquake, Annali di Geofisica, 44, 2, 369-381, 2001.

Nagao, T., Enomoto, Y., Fujinawa, Y., Hata, M., Hayakawa, M., Huang, Q., Izutsu, J., Kushida, Y., Maeda, K., Oike, K., Uyeda, S., and Yoshino, T.: Electromagnetic anomalies associated with 1995 Kobe earthquake, J. Geodynamics, 33, 4-5, 349-359, 2002.

Oike, K. and Ogawa, T.: Observations of electromagnetic radiation related with the occurrence of earthquake, Annu. Rep. Desaster Prev. Res. Inst. Kyoto Univ., 25, B-1, 89-100, 1982.

Oike, K. and Yamada, T.: Relationship between shallow earthquakes and electromagnetic noises in the LF and VLF ranges, in: Electromagnetic phenomena related to earthquake prediction, (Eds) Hayakawa, M. and Fujinawa, Y., Terrapub, Tokyo, 115130, 1994.

Ohnaka, M.: A physical scaling relation between the size of an earthquake and its nucleation zone size, Pure and Applied Geophys., 157, 2259-2282, 2000.

Ohnaka, M. and Mogi, K.: Frequency characteristics of acoustic emission in rocks under uniaxial compression and its relation to the fracturing process to failure, J. Geophys. Res., 87, 38733884, 1982.

Papadopoulos, A.: The Athens, Greece, earthquake $M_{S}=5.9$ of 7 September 1999: An event triggered by the Izmit, Turkey, 17 August 1999 earthquake?, BSSA, 92, 312-321, 2002.

Petrenko, V. and Gluschenkov, O.: Crack velocities in freshwater and saline ice, J. Geophys. Res., 101, B5, 11 541-11 551, 1996.

Ponomarev, A., Zavyalov, A., Smirnov, V., and Lockner, D.: Physical modelling of the formation and evolution of seismically active fault zones, Tectonophysics, 277, 57-81, 1997.

Qian, S., Yian, J., Cao, H., Shi, S., Lu, Z., Li, J., and Ren, K.: Results of observations on seismo-electromagnetic waves at two earthquake experimental areas in China, in: Electromagnetic phenomena related to earthquake prediction, (Eds) Hayakawa, M. and Fujinawa, Y., Terrapub, Tokyo, 205-211, 1994.

Reches, Z. and Lockner, D.: Nucleation and growth of faults in brittle rocks, J. Geophys. Res., 99, 18 159-18 173, 1994.

Shamina, O. (Ed): Model studies of the earthquake source physics, Moscow: Nauka, 191 pp., 1981.

Sornette, D. (Ed): Critical Phenomena in Natural Sciences, Springer, 434 pp., 2000.
Sorokin, V. M. and Chmyrev, V. M.: Modification of the ionosphere by seismic related electric field, in: Atmospheric and Ionospheric Electromagnetic phenomena associated with earthquakes prediction, (Ed) Hayakawa, M., Terrapub, Tokyo, 805-818, 1999.

Swanson, P.: Subcritical crack growth and other time-and-time environment-depending behaviour in rocks, J. Geophys. Res., 89, 4137-4152, 1984.

Tate, J. and Daily, W.: Evidence of electro-seismic phenomena, Phys. Earth Plan. Interior, 57, 1-10, 1989.

Telesca, L., Cuomo, V., and Lapenna, V.: A new approach to investigate the correlation between geoelectrical time fluctuations and earthquakes in a seismic area of southern Italy, Geophys. Res. Lett., 28, 4375-4378, 2001.

Thiel, D.: Short thin-wire antenna to measure the horizontal electric field component at VLF on the Earth's surface, Electronics Letters, 14, 25, 804-805, 1978.

Tzchichholz, F. and Hermann, H. J.: Simulations of pressure fluctuations and accoustic emission in hydraulic fracturing, Phys. Rev. E., 51, 3, 1961-1970, 1995.

Varotsos, P. and Alexopoulos, K.: Physical properties of the variations of the electric field of the Earth preceding earthquakes, I, Tectonophysics, 110, 93-98, 1984a.

Varotsos, P. and Alexopoulos, K.: Physical properties of the variations of the electric field of the Earth preceding earthquakes, II, Tectonophysics, 110, 99-125, 1984b.

Varotsos, P., Lazaridou, M., Eftaxias, K., Antonopoulos, G., Makris, J., and Kopanas, J.: Short-term Earthquake Prediction in Greece by Seismic Electric Signals, in: A Critical Review of VAN: Earthquake prediction from Seismic Electric Signals, (Ed) Ligthhill, Sir J., World Scientific Publishing Co., Singapore, 2976, 1996.

Vershinin, E. F., Buzevich, A. V., Yomoto, K., Saita, K., and Tanaka, Y.: Correlations of Seismic Activity with Electromagnetic Emissions and Variations in Kamacchatka Region, in: Atmospheric and Ionospheric Electromagnetic Phenomena Associated with Earthquakes, (Ed) Hayakawa, M., Terrapub, Tokyo, 513-517, 1999.

Uyeda, S.: Introduction to the VAN method of earthquake prediction, in: A critical review of VAN-Earthquake Prediction from Seismic Electric Signals, (Ed) Lighthill, Sir J., World Scientific Publishing Co., Singapore, 3-28, 1996.

Uyeda, S., Nagao, T., Orihara, Y., Yamaguchi, T., and Takahashi, I.: Geoelectric potential changes: Possible precursors to earthquakes in Japan, Proc. Nat. Acad. Sci., 97, 4561-4566, 2000.

Yamada, I., Masuda, K., and Mizutani, H.: Electromagnetic and acoustic emission associated with rock fracture, Physics of the Earth and Planetary Interiors, 57, 157-168, 1989.

Warwick, J., Stoker, C., and Meyer, T.: Radio emission associated with rock fracture: possible application to the great Chilean earthquake of 22 May 1960, J. Geophys. Res., 87, 2851-2859, 1982.

Wu, F. and Thomsen, L.: Microfracturing and deformation of westerly granite at the creep condition, Int. J. Rock Mech. Mining Sci. Geomech. Abst., 12, 167-173, 1975. 